Владикавказский математический журнал 2020, Том 22, Выпуск 4, С. 28-44

УДК 517.955 .8

DOI 10.46698/s0301-1959-8380-s

\title{
ВОССТАНОВЛЕНИЕ БЫСТРО ОСЦИЛЛИРУЮЩЕЙ ПРАВОЙ ЧАСТИ ВОЛНОВОГО УРАВНЕНИЯ ПО ЧАСТИЧНОЙ АСИМПТОТИКЕ РЕШЕНИЯ\#
}

\author{
П. В. Бабич ${ }^{1}$ В. Б. Левенштам ${ }^{1}$ \\ ${ }^{1}$ Математический институт им. В. А. Стеклова \\ Россия, 119991, Москва, ул. Губкина, 8 \\ E-mail: xblahblahc@gmail.com, vlevenshtam@yandex.ru
}

Работа посвящается 75-летию Семена Самсоновича Кутателадзе

\begin{abstract}
Аннотация. В работе исследуется однородная начально-краевая задача для одномерного волнового уравнения с неизвестной быстро осциллирующей по времени правой частью. Последняя представлена произведением двух функций, одна из которых зависит от пространственной переменной, а вторая - от временной и быстрой временной переменных. Рассматриваются четыре различных случая, в двух из которых одна из функций-сомножителей известна, а в двух других - обе функции неизвестны. В каждом случае поставлены и решены обратные задачи о восстановлении неизвестных функций по некоторым сведениям о частичных асимптотиках решений исходной задачи с известными данными. Указанные сведения состоят в основном в задании значений определенных коэффициентов асимптотик в некоторых точках пространства и/или времени. Использование дополнительных условий (условий переопределения) в таком виде говорит о коренном отличии данных постановок обратных задач от классики, где дополнительные условия ставятся на точные решения. Построение асимптотики решения исходной задачи при этом подходе играет роль прямой задачи. Указанный подход к обратным задачам с быстро осциллирующими по времени данными авторы данной статьи развивают несколько последних лет.
\end{abstract}

Ключевые слова: одномерное волновое уравнение, быстро осциллирующая правая часть, асимптотика решения, обратные задачи о восстановлении правой части.

Mathematical Subject Classification (2010): 35L05, 35L20, 35R30.

Образец цитирования: Бабич П. В., Левенштам В. Б. Восстановление быстро осциллирующей правой части волнового уравнения по частичной асимптотике решения // Владикавк. мат. журн.2020.-T. 22, вып. 4.-C. 28-44. DOI: 10.46698/s0301-1959-8380-s.

\section{Введение}

В работе рассматривается начально-краевая задача для одномерного волнового уравнения с неизвестной быстро осциллирующей по времени правой частью. Исследуется задача о восстановлении этой правой части по тем или иным сведениям (дополнительным условиям) о нескольких первых коэффициентах асимптотики решения. Ранее аналогичный вопрос был исследован нами для одномерного уравнения теплопроводности $[1,2]$. Теории обратных задач посвящен целый ряд монографий (см., например [3-6]) и большое число статей. В статьях [7-9], например, решены различные задачи о восстановлении

\# Работа выполнена при финансовой поддержке Российского научного фонда, проект № 20-11-20141. (c) 2020 Бабич П. В., Левенштам В. Б. 
неизвестных правых частей одномерных уравнения теплопроводности и волнового уравнения. В [3-9] быстрые осцилляции данных задачи отсутствуют. В данной работе задача о восстановлении правой части вида $f(x, t) r(t, \omega t)$, где сомножитель $r(t, \tau)$ периодичен по $\tau$, волнового уравнения с большим параметром $\omega$ поставлена и решена в следующих четырех случаях:

1) не известна функция $r(t, \tau)$;

2) не известна функция $f(x, t) \equiv f(x)$;

$3)$ в паре $f, r$ известно лишь среднее $r_{0}$ функции $r(t, \tau)$ по $\tau$;

4) не известны обе функции $-f$ и $r$.

Каждая из этих задач снабжена дополнительными условиями, которые относятся к нескольким первым коэффициентам асимптотики решения. В этом состоит основное отличие данных постановок обратных задач от классических, где дополнительные условия относятся к точному решению. В заключение отметим, что результаты, аналогичные теоремам 1, 2 раздела 1 данной работы, относящимся к задаче 1), установлены нами и для многомерных гиперболических уравнений [10]. При этом доказательство этих результатов в [10] базируется на важной работе [11], а соответствующие одномерные результаты данной работы доказаны в разделе 1 с помощью непосредственного использования техники одномерных рядов Фурье, так что они изложены в замкнутом виде.

\section{1. Неизвестный сомножитель источника зависит только от временной переменной}

1.1. Прямая задача. Символом П обозначим замкнутый плоский прямоугольник:

$$
\Pi=\{(x, t): 0 \leqslant x \leqslant \pi ; 0 \leqslant t \leqslant T\}, \quad T>0 .
$$

Рассмотрим в П начально-краевую задачу для волнового уравнения с большим параметpom $\omega$ :

$$
\begin{gathered}
\frac{\partial^{2} u}{\partial t^{2}}=\frac{\partial^{2} u}{\partial x^{2}}+f(x, t) r(t, \omega t), \quad(x, t) \in \Pi \\
\left.u\right|_{t=0}=0,\left.\quad \frac{\partial u}{\partial t}\right|_{t=0}=0 \\
\left.u\right|_{x=0, \pi}=0 .
\end{gathered}
$$

Здесь функция $f(x, t)$ определена на П и существуют непрерывные на П функции

$$
f, f_{x^{2}}^{\prime \prime}, f_{x^{2} t}^{\prime \prime \prime}, f_{t}^{\prime}, f_{t^{2}}^{\prime \prime}, f_{t^{3}}^{\prime \prime \prime}
$$

обращающиеся в нуль при $x=0, x=\pi$, причем каждая из функций (4) имеет две непрерывные на П производные по $x$. Ясно, что справедливость условий равенства нулю функций (4) при $x=0, \pi$ достаточно проверять лишь для функций $f$ и $f_{x^{2}}^{\prime \prime}$.

Пусть $Q$ - полуполоса: $Q=\{(t, \tau) \in[0, T] \times[0, \infty)\}$. Относительно функции $r(t, \tau)$ будем предполагать, что она определена и непрерывна на множестве $Q$, а также $2 \pi$-периодична по $\tau$. Обозначим через $r_{0}(t)$ ее среднее по $\tau$, т. е. плавную часть:

$$
r_{0}(t)=\langle r(t, \tau)\rangle_{\tau}=\frac{1}{2 \pi} \int_{0}^{2 \pi} r(t, \tau) d \tau,
$$


а через $r_{1}(t, \tau)$ - быструю часть:

$$
r_{1}(t, \tau)=r(t, \tau)-r_{0}(t) \equiv\left\{r_{1}(t, \tau)\right\}_{\tau}
$$

Будем предполагать, что функции $\frac{\partial^{k} r_{1}}{\partial t^{k}} \in C(Q), k=0,1,2,3$. Функцию $r(t, \tau)$, удовлетворяющую указанным в этом абзаце условиям, будем для краткости называть функцией класса (А).

Введем некоторые обозначения, которыми будем пользоваться в дальнейшем:

$$
\begin{gathered}
f_{n}(t)=\frac{1}{\pi} \int_{0}^{\pi} f(s, t) \sin n s d s, \quad f_{2, n}(t)=\frac{1}{\pi} \int_{0}^{\pi} \frac{\partial^{2} f(s, t)}{\partial s^{2}} \sin n s d s \\
\rho_{0}(t, \tau)=\int_{0}^{\tau}\left(\int_{0}^{p} r_{1}(t, s) d s-\left\langle\int_{0}^{\tau} r_{1}(t, s) d s\right\rangle\right) d p \\
\left.-\left\langle\int_{0}^{\tau}\left(\int_{0}^{p} r_{1}(t, s) d s-\left\langle\int_{0}^{\tau} r_{1}(t, s) d s\right\rangle\right) d p\right\rangle_{\tau}^{p} \equiv\left\{\int_{0}^{\tau}\left\{\int_{0}^{p} r_{1}(t, s) d s\right\}_{p}\right\}_{\tau}\right\}_{\tau} .
\end{gathered}
$$

Решение задачи (1)-(3) представим в виде:

$$
u_{\omega}(x, t)=U_{\omega}(x, t)+W_{\omega}(x, t), \quad \omega \gg 1,
$$

где

$$
\begin{gathered}
U_{\omega}(x, t)=u_{0}(x, t)+\omega^{-1} u_{1}(x, t)+\omega^{-2}\left[u_{2}(x, t)+v_{2}(x, t, \omega t)\right], \quad \omega \gg 1, \\
u_{0}(x, t)=\sum_{n=1}^{\infty} \frac{\sin n x}{n} \int_{0}^{t} \sin n(t-s) f_{n}(s) r_{0}(s) d s, \\
u_{1}(x, t)=-\sum_{n=1}^{\infty} f_{n}(0) \rho_{0 t}^{\prime}(0,0) \frac{\sin n x}{n} \sin n t, \\
v_{2}(x, t, \tau)=f(x, t) \rho_{0}(t, \tau), \\
u_{2}(x, t)=-\sum_{n=1}^{\infty} f_{n}(0) \rho_{0}(0,0) \cos n t \sin n x \\
+\sum_{n=1}^{\infty}\left(f_{n t}^{\prime}(0) \rho_{0}(0,0)+f_{n}(0) \rho_{0 t}^{\prime}(0,0)\right) \sin n t \frac{\sin n x}{n} .
\end{gathered}
$$

Теорема 1. Решение $u_{\omega}(x, t)$ задачи (1)-(3) представимо в виде (8)-(13), где функция $W_{\omega}$ удовлетворяет соотношению

$$
\left\|W_{\omega}(x, t)\right\|_{C(\Pi)}=o\left(\omega^{-2}\right), \quad \omega \rightarrow \infty .
$$

1.2. Обратная задача 1. Предположим, что в задаче (1)-(3) фигурирует та же, что в п.1.1, функция $f(x, t)$, а функция $r(t, \tau)$ класса $(\mathrm{A})$ неизвестна. Пусть задана точка 
$x_{0} \in(0, \pi)$ такая, что $f\left(x_{0}, t\right) \neq 0, t \in[0, T]$, и функции $\varphi_{0}(t)$ и $\chi(t, \tau)$, принадлежащие следующим классам:

$$
\varphi_{0} \in C^{2}([0, T]), \quad \varphi_{0}(0)=0, \quad \varphi_{0}^{\prime}(0)=0
$$

$\chi(t, \tau)$ - непрерывная в $Q, 2 \pi$-периодическая по $\tau$ с нулевым средним функция, производные которой $\frac{\partial^{k+2} \chi}{\partial t^{k} \partial \tau^{2}}, k=0,1,2,3$, принадлежат $C(Q)$. Введем теперь функции $\varphi_{1}(t)$ и $\varphi_{2}(t):$

$$
\varphi_{1}(t)=-\sum_{n=1}^{\infty} f_{n}(0) \rho_{0 t}^{\prime}(0,0) \sin n t \frac{\sin n x_{0}}{n}
$$

$$
\begin{aligned}
\varphi_{2}(t)=-\sum_{n=1}^{\infty} f_{n}(0) \rho_{0}(0,0) \cos n t & \sin n x_{0} \\
& +\sum_{n=1}^{\infty}\left(f_{n t}^{\prime}(0) \rho_{0}(0,0)+f_{n}(0) \rho_{0 t}^{\prime}(0,0)\right) \sin n t \frac{\sin n x_{0}}{n}
\end{aligned}
$$

где $\rho_{0}(t, \tau)$ задается равенством $(7) \mathrm{c}$

$$
r_{1}(t, \tau)=\frac{1}{f\left(x_{0}, t\right)} \frac{\partial^{2}}{\partial \tau^{2}} \chi(t, \tau)
$$

Обратная задача 1 заключается в определении функции $r(t, \tau)$ класса $(\mathrm{A})$, при которой для решения $u_{\omega}(x, t)$ задачи $(1)-(3)$ (функция $f(x, t)$ задана в п.1.1) выполняется асимптотическое равенство

$$
\left\|u_{\omega}\left(x_{0}, t\right)-\left[\varphi_{0}(t)+\frac{1}{\omega} \varphi_{1}(t)+\frac{1}{\omega^{2}}\left(\varphi_{2}(t)+\chi(t, \omega t)\right)\right]\right\|_{C([0, T])}=o\left(\omega^{-2}\right), \quad \omega \rightarrow \infty .
$$

Здесь точка $x_{0}$ и функции $\varphi_{0}, \varphi_{1}, \varphi_{2}, \chi$ удовлетворяют условиям, указанным в предыдущей части этого пункта.

Теорема 2. Для любых функций $\chi, \varphi_{0}$ и точки $x_{0}$, удовлетворяющих сформулированным выше условиям, обратная задача 1 однозначно разрешима, т. е. найдется единственная функция $r$ класса (A), при которой решение $u_{\omega}(x, t)$ задачи $(1)-(3)$ удовлетворяет соотношению (18).

ЗАмЕчАниЕ. Функция $r_{0}(t)=\langle r(t, \tau)\rangle_{\tau}$ находится из уравнения Вольтерра второго рода, а $r_{1}(t, \tau)$ определяется равенством (17).

\section{3. Доказательство основных результатов.}

$\triangleleft$ ДоказатеЛЬСтво теоремы 1 . Решение задачи (1)-(3) запишем в виде суммы

$$
u_{\omega}(x, t)=u_{0}(x, t)+\omega^{-1} u_{1}(x, t)+\omega^{-2}\left[u_{2}(x, t)+v_{2}(x, t, \omega t)\right]+\omega^{-3} v_{3}(x, t, \omega t)+Z_{\omega}(x, t),
$$

где функции $u_{i}, v_{i}$ и $Z_{\omega}$ будут определены ниже. 
Подставив последнее представление $u_{\omega}$ в равенства (1)-(3), придем к соотношениям

$$
\left\{\begin{array}{l}
\frac{\partial^{2} u_{0}}{\partial t^{2}}+\frac{\partial^{2} v_{2}}{\partial \tau^{2}}+\omega^{-1}\left[\frac{\partial^{2} u_{1}}{\partial t^{2}}+2 \frac{\partial^{2} v_{2}}{\partial \tau \partial t}+\frac{\partial^{2} v_{3}}{\partial \tau^{2}}\right]+\omega^{-2}\left[\frac{\partial^{2} u_{2}}{\partial t^{2}}+\frac{\partial^{2} v_{2}}{\partial t^{2}}+2 \frac{\partial^{2} v_{3}}{\partial \tau \partial t}\right] \\
\quad+\omega^{-3} \frac{\partial^{2} v_{3}}{\partial t^{2}}+\frac{\partial^{2} Z_{\omega}}{\partial t^{2}}=\frac{\partial^{2} u_{0}}{\partial x^{2}}+\omega^{-1} \frac{\partial^{2} u_{1}}{\partial x^{2}}+\omega^{-2}\left[\frac{\partial^{2} u_{2}}{\partial x^{2}}+\frac{\partial^{2} v_{2}}{\partial x^{2}}\right]+\omega^{-3} \frac{\partial^{2} v_{3}}{\partial x^{2}} \\
\quad+\frac{\partial^{2} Z_{\omega}}{\partial x^{2}}+f(x, t) r(t, \tau) ; \\
{\left[u_{0}+\omega^{-1} u_{1}+\omega^{-2}\left(u_{2}+v_{2}\right)+\omega^{-3} v_{3}+Z_{\omega}\right]_{t, \tau=0}=0 ;} \\
{\left[\frac{\partial u_{0}}{\partial t}+\omega^{-1}\left(\frac{\partial u_{1}}{\partial t}+\frac{\partial v_{2}}{\partial \tau}\right)+\omega^{-2}\left(\frac{\partial u_{2}}{\partial t}+\frac{\partial v_{2}}{\partial t}+\frac{\partial v_{3}}{\partial \tau}\right)+\omega^{-3} \frac{\partial v_{3}}{\partial t}+\frac{\partial Z_{\omega}}{\partial t}\right]_{t, \tau=0}=0} \\
{\left[u_{0}+\omega^{-1} u_{1}+\omega^{-2}\left(u_{2}+v_{2}\right)+\omega^{-3} v_{3}+Z_{\omega}\right]_{x=0, \pi}=0, \tau=\omega t .}
\end{array}\right.
$$

Приравняем формально в последних равенствах коэффициенты при явно выписанных степенях $\omega^{-k}, k=0,1,2,3$. Применяя к полученным уравнениям операцию усреднения $\langle\ldots\rangle$ по $\tau=\omega t$, придем к задачам

$$
\begin{gathered}
\quad\left\{\begin{array}{l}
\frac{\partial^{2} u_{0}}{\partial t^{2}}=\frac{\partial^{2} u_{0}}{\partial x^{2}}+f(x, t) r_{0}(t) \\
\left.u_{0}\right|_{t=0}=0,\left.\frac{\partial u_{0}}{\partial t}\right|_{t=0}=0 \\
\left.u_{0}\right|_{x=0, \pi}=0,
\end{array}\right. \\
\qquad \begin{array}{l}
\frac{\partial^{2} v_{2}}{\partial \tau^{2}}=f(x, t) r_{1}(t, \tau) ; \\
v_{2}(x, t, \tau+2 \pi)=v_{2}(x, t, \tau) \\
\left\langle v_{2}(x, t, \tau)\right\rangle_{\tau}=0
\end{array} \\
\left\{\begin{array}{l}
\frac{\partial^{2} u_{1}}{\partial t^{2}}=\frac{\partial^{2} u_{1}}{\partial x^{2}} \\
\left.u_{1}\right|_{t=0}=0,\left.\frac{\partial u_{1}}{\partial t}\right|_{t=0}=-\left.\frac{\partial v_{2}}{\partial \tau}\right|_{t, \tau=0} \\
\left.u_{1}\right|_{x=0, \pi}=0,
\end{array}\right. \\
\left\{\begin{array}{l}
\frac{\partial^{2} v_{3}}{\partial \tau^{2}}=-2 \frac{\partial^{2} v_{2}}{\partial \tau \partial t} ; \\
v_{3}(x, t, \tau+2 \pi)=v_{3}(x, t, \tau) \\
\left\langle v_{3}(x, t, \tau)\right\rangle_{\tau}=0,
\end{array}\right. \\
\left\{\begin{array}{l}
\frac{\partial^{2} u_{2}}{\partial t^{2}}=\frac{\partial^{2} u_{2}}{\partial x^{2}} \\
\left.u_{2}\right|_{t=0}=-\left.v_{2}\right|_{t, \tau=0},\left.\frac{\partial u_{2}}{\partial t}\right|_{t=0}=-\left[\frac{\partial v_{3}}{\partial \tau}+\frac{\partial v_{2}}{\partial t}\right]_{t, \tau=0} \\
\left.u_{2}\right|_{x=0, \pi}=0,
\end{array}\right.
\end{gathered}
$$

В силу (20)-(24) функции $u_{0}, u_{1}, u_{2}, v_{2}$ имеют вид $(10),(11),(12)$ и (13) соответственно, что устанавливается с помощью элементарного применения рядов Фурье. Из системы (23) находим

$$
\begin{aligned}
v_{3}(x, t, \tau) & =2 f_{t}^{\prime}(x, t) \rho_{1}(t, \tau)+2 f(x, t) \rho_{1 t}^{\prime}(t, \tau), \\
\rho_{1}(t, \tau) & =\left\langle\int_{0}^{\tau} \rho_{0}(t, s) d s\right\rangle_{\tau}-\int_{0}^{\tau} \rho_{0}(t, s) d s .
\end{aligned}
$$


Из (19) с учетом (20)-(24) следует, что функция $Z_{\omega}(x, t)$ является решением задачи

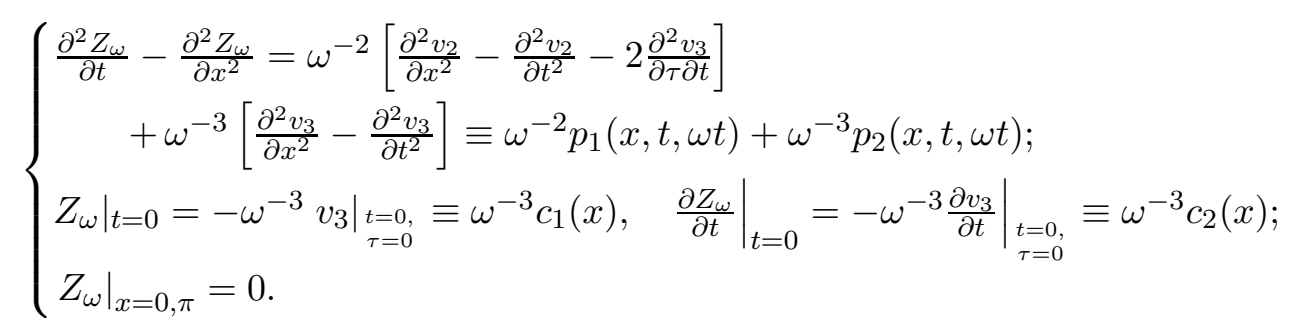

При этом согласно $(12),(25)$ функции $p_{i}(x, t, \tau) 2 \pi$-периодичны по $\tau$ с нулевым средним и, кроме того, $\left.p_{i}(x, t, \tau)\right|_{x=0, \pi}=0,\left.c_{i}(x)\right|_{x=0, \pi}=0, i=1,2$.

Решение задачи (26) имеет вид

$$
\begin{aligned}
Z_{\omega}(x, t)=\sum_{n=1}^{\infty} \sin n x\left[\int _ { 0 } ^ { t } \frac { \operatorname { s i n } n ( t - s ) } { n } \left(\omega^{-2} p_{1, n}(s, \omega s)\right.\right. & \left.+\omega^{-3} p_{2, n}(s, \omega s)\right) d s \\
& \left.+\omega^{-3} c_{1, n} \cos n t+\omega^{-3} \frac{c_{2, n}}{n} \sin n t\right]
\end{aligned}
$$

где

$$
p_{i, n}(t, \tau)=\frac{1}{\pi} \int_{0}^{\pi} p_{i}(s, t, \tau) \sin n s d s, \quad c_{i, n}=\frac{1}{\pi} \int_{0}^{\pi} c_{i}(s) \sin n s d s, \quad i=1,2 .
$$

Поскольку $W_{\omega}(x, t)=Z_{\omega}(x, t)+\omega^{-3} v_{3}(x, t, \omega t)$, то теорема 1 будет доказана, если мы установим асимптотическое равенство

$$
\left\|Z_{\omega}\right\|_{C(\bar{\Pi})}=o\left(\omega^{-2}\right), \quad \omega \rightarrow \infty .
$$

Заметим, что благодаря требованиям, предъявленным к $f$ и $r$, функции $p_{2}(x, t, \tau), c_{1}(x)$, $c_{2}(x)$ непрерывны, а ряды

$$
\begin{gathered}
\sum_{n=1}^{\infty} \frac{\sin n x}{n} \int_{0}^{t} \sin n(t-s) p_{i, n}(s, \omega s) d s, \quad i=1,2, \\
\sum_{n=1}^{\infty} c_{1, n} \sin n x \cos n t, \quad \sum_{n=1}^{\infty} \frac{c_{2, n}}{n} \sin n x \sin n t
\end{gathered}
$$

сходятся равномерно относительно $(x, t) \in \bar{\Pi}$. Отсюда следует, что нам теперь достаточно доказать при любом фиксированном $n_{0} \in \mathbb{N}$ оценку

$$
\left\|\sum_{n=1}^{n_{0}} \frac{\sin n x}{n} \int_{0}^{t} \sin n(t-s) p_{1, n}(s, \omega s) d s\right\|_{C([0, T])}=o(1), \quad \omega \rightarrow \infty .
$$

Проведем ее в два этапа. Пусть $\varepsilon-$ произвольное положительное число. На первом этапе подберем достаточно малое число $t_{0}>0$, при котором для всех $x \in[0, \pi], t \in\left[0, t_{0}\right]$, и $\omega>0$ справедлива оценка

$$
\left|\sum_{n=1}^{n_{0}} \frac{\sin n x}{n} \int_{0}^{t} \sin n(t-s) p_{1, n}(s, \omega s) d s\right|<\varepsilon
$$


На втором этапе участок $[0, t], t \in\left[t_{0}, T\right]$, разобьем на $m$ равных частей $\left[t_{j}, t_{j+1}\right)$, $j=0,1, \ldots, m-1$, и воспользуемся равенством

$$
\begin{gathered}
\int_{0}^{t} \sin n(t-s) p_{1, n}(s, \omega s) d s \\
=\sum_{j=0}^{m-1}\left[\int_{t_{j}}^{t_{j+1}} \sin n(t-s) p_{1, n}(s, \omega s) d s-\int_{t_{j}}^{t_{j+1}} \sin n\left(t-t_{j}\right) p_{1, n}\left(t_{j}, \omega s\right) d s\right] \\
+\sum_{j=0}^{m-1} \int_{t_{j}}^{t_{j+1}} \sin n\left(t-t_{j}\right) p_{1, n}\left(t_{j}, \omega s\right) d s=S_{1}+S_{2} .
\end{gathered}
$$

Выберем $m$ столь большим, что при всех $\omega>0$

$$
\left|S_{1}\right|<\frac{\varepsilon}{2 n_{0}} \text {. }
$$

Далее, в силу равенства

$$
\int_{t_{j}}^{t_{j+1}} \sin n\left(t-t_{j}\right) p_{1, n}\left(t_{j}, \omega s\right) d s=\sin n\left(t-t_{j}\right)\left[\frac{1}{\omega} \int_{0}^{\omega t_{j+1}} p_{1, n}\left(t_{j}, \tau\right) d \tau-\frac{1}{\omega} \int_{0}^{\omega t_{j}} p_{1, n}\left(t_{j}, \tau\right) d \tau\right]
$$

и того факта, что функция $p_{1, n}(s, \tau)$ имеет нулевое среднее по второй переменной, найдем такое $\omega_{0}>0$, что при $\omega>\omega_{0}$

$$
\left|S_{2}\right|<\frac{\varepsilon}{2 n_{0}} .
$$

Из соотношений (28)-(30) следует (27). Теорема 1 доказана. $\triangleright$

$\triangleleft$ ДоКАЗАТЕЛЬСТво теОРемы 2. В силу теоремы 1 решение задачи (1)-(3) при заданной функции $r(t, \tau)$ класса (А) представимо в виде $(8),(9)-(13)$, где

$$
\left\|W_{\omega}\right\|_{C(\Pi)}=o\left(\omega^{-2}\right), \quad \omega \rightarrow \infty .
$$

Предположим, что $r(t, \tau)$ является решением обратной задачи, и $u_{\omega}$ - отвечающее ему решение задачи (1)-(3). В силу соотношений $(14),(18)$ равномерно относительно $t \in[0, T]$

$$
\begin{aligned}
u_{0}\left(x_{0}, t\right)+\omega^{-1} u_{1}\left(x_{0}, t\right) & +\omega^{-2}\left[u_{2}\left(x_{0}, t\right)+v_{2}\left(x_{0}, t, \omega t\right)\right] \\
& =\varphi_{0}(t)+\frac{1}{\omega} \varphi_{1}(t)+\frac{1}{\omega^{2}}\left(\varphi_{2}(t)+\chi(t, \omega t)\right)+o\left(\omega^{-2}\right), \quad \omega \gg 1 .
\end{aligned}
$$

Приравняв в (31) коэффициенты при одинаковых степенях $\omega$ и используя операцию усреднения по $\tau$, приходим к соотношениям

$$
u_{0}\left(x_{0}, t\right)=\varphi_{0}(t), u_{1}\left(x_{0}, t\right)=\varphi_{1}(t), \quad u_{2}\left(x_{0}, t\right)=\varphi_{2}(t), v_{2}\left(x_{0}, t, \tau\right)=\chi(t, \tau),
$$

где $t \in[0, T], \tau \in[0, \infty)$. Дифференцируя первое из этих равенств дважды по $t$, а последнее дважды по $\tau$, получим

$$
\begin{gathered}
\frac{\partial^{2}}{\partial t^{2}} u_{0}\left(x_{0}, t\right)=\varphi_{0}^{\prime \prime}(t), \\
\frac{\partial^{2}}{\partial \tau^{2}} v_{2}\left(x_{0}, t, \tau\right)=\frac{\partial^{2}}{\partial \tau^{2}} \chi(t, \tau) .
\end{gathered}
$$


Согласно п.1.1 $\frac{\partial^{2} v_{2}(x, t, \tau)}{\partial \tau^{2}}=f(x, t) r_{1}(t, \tau)$. Отсюда с учетом (33) находим

$$
f\left(x_{0}, t\right) r_{1}(t, \tau)=\frac{\partial^{2}}{\partial \tau^{2}} \chi(t, \tau) .
$$

В силу п.1.1 функция $u_{0}(x, t)$ удовлетворяет равенству $(10)$, так что

$$
\frac{\partial^{2}}{\partial t^{2}} u_{0}\left(x_{0}, t\right)=f\left(x_{0}, t\right) r_{0}(t)+\int_{0}^{t} K(t, s) r_{0}(s) d s,
$$

где

$$
K(t, s)=-\sum_{n=1}^{\infty} n f_{n}(s) \sin n(t-s) \sin n x_{0} .
$$

Отсюда следует, что функция $K(t, s)$ непрерывна. От равенств $(32),(35)$ приходим к уравнению Вольтерра второго рода

$$
f\left(x_{0}, t\right) r_{0}(t)+\int_{0}^{t} K(t, s) r_{0}(s) d s=\varphi_{0}^{\prime \prime},(t)
$$

из которого однозначно определяется непрерывная функция $r_{0}(t)$. Из уравнения (34) функция $r_{1}$ также определяется единственным образом:

$$
r_{1}(t, \tau)=\frac{1}{f\left(x_{0}, t\right)} \frac{\partial^{2}}{\partial \tau^{2}} \chi(t, \tau)
$$

которая в силу условий, наложенных на функцию $\chi$, удовлетворяет указанным в п.1.1 условиям.

Поскольку найденная функция $r(t, \tau)=r_{0}(t)+r_{1}(t, \tau)$ является функцией класса (А), то для нее справедлива теорема 1 , так что решение задачи (1)-(3) представимо в виде (8)-(13). Покажем, что для функции $u_{\omega}(x, t)$ будет выполнено условие (18). Для этого достаточно установить равенства:

$$
u_{0}\left(x_{0}, t\right)=\varphi_{0}(t), \quad u_{1}\left(x_{0}, t\right)=\varphi_{1}(t), \quad u_{2}\left(x_{0}, t\right)=\varphi_{2}(t), \quad v_{2}\left(x_{0}, t, \tau\right)=\chi(t, \tau) .
$$

В силу соотношений $(35),(36) \frac{\partial^{2}}{\partial t^{2}} u_{0}\left(x_{0}, t\right)=\varphi_{0}^{\prime \prime}(t)$. Поскольку $u_{0}\left(x_{0}, 0\right)=\varphi_{0}(0)=0$, $\left.\frac{\partial u_{0}\left(x_{0}, t\right)}{\partial t}\right|_{t=0}=\varphi_{0}^{\prime}(0)=0$, то $u_{0}\left(x_{0}, t\right)=\varphi_{0}(t)$. Выразим $r_{1}$ из (34) и подставим в $(21)$. Положим затем $x=x_{0}$ и учтем, что функции $\chi(t, \tau)$ и $v_{2}(x, t, \tau) 2 \pi$-периодические $\mathrm{c}$ нулевым средним по $\tau$. В результате получим $v_{2}\left(x_{0}, t, \tau\right)=\chi(t, \tau)$. Заметим, наконец, что согласно (11) и $(15) u_{1}\left(x_{0}, t\right)=\varphi_{1}(t)$, а согласно $(13)$ и $(16) u_{2}\left(x_{0}, t\right)=\varphi_{2}(t)$. Теорема 2 доказана. $\triangleright$

\section{2. Неизвестный сомножитель источника зависит от пространственной переменной}

2.1. Прямая задача. Пусть П и $\mathrm{Q}$ - те же множества, что в п. 1.1. Рассмотрим задачу (1)-(3) с $f(x, t) \equiv f(x)$. Предположим, что $f \in C^{2}([0, \pi]), f(0)=f(\pi)=0$, 
а непрерывная функция $r(t, \tau)=r_{0}(t)+r_{1}(t, \tau)-2 \pi$-периодична по $\tau, r_{0} \in C([0, T])-$ ее среднее, $r_{1} \in C_{2 \pi}^{\alpha, 0}(Q), \alpha \in(0,1)^{*}$. Введем обозначение:

$$
u_{0}(x, t)=\sum_{n=1}^{\infty} f_{n} \sin n x \int_{0}^{t} \frac{\sin n(t-s)}{n} r_{0}(s) d s \equiv \sum_{n=1}^{\infty} f_{n} \Lambda_{n}(t) \sin n x,
$$

где $f_{n}$ - коэффициенты разложения функции $f(x)$ в ряд Фурье по синусам (см. п.1.1).

Теорема 3. Справедлива асимптотическая формула

$$
\left\|u_{\omega}-u_{0}\right\|_{C(\Pi)}=o(1), \quad \omega \rightarrow \infty,
$$

где $u_{\omega}$ - решение задачи (1)-(3).

2.2. Обратная задача 2. Рассмотрим задачу (1)-(3). Будем считать, что функция $r(t, \tau)$ известна, удовлетворяет условиям п.2.1 и дополнительно $r_{0} \in C^{2}([0, T])$, а функция $f(x, t) \equiv f(x)$, удовлетворяющая условиям п.2.1, неизвестна.

Справедлива следующая лемма, в которой $\Lambda_{n}(t), n \in \mathbb{N}, t \in[0, T],-$ те же функции, что в формуле (37).

Лемма 1. Если существует число $t_{0} \in(0, T]$, при котором $\left|r_{0}\left(t_{0}\right)\right|>\left|r_{0}(0)\right|$, то найдутся числа $c_{0}>0$ и $n_{0} \in \mathbb{N}$ такие, что при $n \geqslant n_{0}$ справедливы оценки $\Lambda_{n}\left(t_{0}\right)>\frac{c_{0}}{n^{2}}$.

Если $r_{0}=$ const $\neq 0$ и $t_{0,1}=2 \pi \frac{l_{0}}{m_{0}}$, где $l_{0}, m_{0} \in \mathbb{N}$ - взаимно просты, то найдутся числа $c_{1}>0$ и $n_{1} \in \mathbb{N}$, при которых для всех $n \geqslant n_{1}, n \neq s m_{0}, s \in \mathbb{N}$, имеют место оценки $\Lambda_{n}\left(t_{0,1}\right)>\frac{c_{0}}{n^{2}}$.

ЗАмечАниЕ. При $r_{0} \equiv 0$, очевидно, $\Lambda_{n}(t) \equiv 0$ при всех $n \in \mathbb{N}$.

Обозначим через $M_{0}$ множество индексов $n \in \mathbb{N}$ таких, что $\Lambda_{n}\left(t_{0}\right)=0$.

Задачу (1)-(3) с неизвестной функцией $f$ дополним заданием некоторой функции

$$
\psi \in C^{5}([0, \pi]), \quad \psi^{(2 j)}(0)=\psi^{(2 j)}(\pi)=0, \quad j=0,1,2 .
$$

Обратная задача 2 состоит в нахождении функции $f$, удовлетворяющей условиям п.2.1, при которой для решения $u_{\omega}(x, t)$ задачи $(1)-(3)$ выполнено соотношение

$$
\left\|u_{\omega}\left(x, t_{0}\right)-\psi(x)\right\|_{C([0, \pi])}=o(1), \quad \omega \rightarrow \infty .
$$

Теорема 4. Пусть существует $t_{0}$ такое, что $\left|r_{0}\left(t_{0}\right)\right|>\left|r_{0}(0)\right|$. Тогда при $M_{0}=\emptyset$ обратная задача однозначно разрешима, и при этом $f_{n}=\frac{\psi_{n}}{\Lambda_{n}}, n \in \mathbb{N}$. Если же $M_{0} \neq \emptyset$, то она разрешима тогда и только тогда, когда $\psi_{n}=0, n \in M_{0}$, и при этом $f_{n}=\frac{\psi_{n}}{\Lambda_{n}}, n \notin M_{0}$, $f_{n}$ - любое число при $n \in M_{0}$.

\section{3. Доказательство основных результатов.}

$\triangleleft$ ДОКАЗАТЕЛЬСТво ТЕОРЕМЫ 3. Рассмотрим функцию

$$
\begin{gathered}
W_{\omega}(x, t)=u_{\omega}(x, t)-u_{0}(x, t)=\sum_{n=1}^{\infty} f_{n} \sin n x \int_{0}^{t} \frac{\sin n(t-s)}{n} r_{1}(s, \omega s) d s \\
=\sum_{n=1}^{n_{0}} f_{n} \sin n x \int_{0}^{t} \frac{\sin n(t-s)}{n} r_{1}(s, \omega s) d s+\sum_{n=n_{0}+1}^{\infty} f_{n} \sin n x \int_{0}^{t} \frac{\sin n(t-s)}{n} r_{1}(s, \omega s) d s \\
\equiv S_{\omega, 1}+S_{\omega, 2}, \quad n_{0} \in \mathbb{N} .
\end{gathered}
$$

${ }^{*}$ Символом $C_{2 \pi}^{\alpha, 0}(Q)$ обозначено обычное банахово пространство непрерывных на множестве $Q$ функций $v(t, \tau)) 2 \pi$-периодичных по $\tau$, удовлетворяющих равномерно относительно $(t, \tau) \in Q$ условию Гёльдера по $t$ с показателем $\alpha$ и снабженных естественной нормой. 
Пусть $\varepsilon-$ произвольное положительное число. Заметим, что в силу условий, наложенных в п.2.1 на $f, r_{1}$, ряд, представляющий $W_{\omega}$, сходится абсолютно и равномерно относительно $(x, t) \in \Pi, \omega>0$. Учитывая это и используя неравенство Коши - Буняковского, подберем $n_{0}$ столь большим, что при всех $\omega>0,(x, t) \in[0, \pi] \times[0, T]$

$$
\left|S_{\omega, 2}\right|<\frac{\varepsilon}{2}
$$

Далее выберем число $t_{0}>0$ столь малым, что при всех $(x, t) \in[0, \pi] \times\left[0, t_{0}\right]$ и $\omega>0$

$$
\left\|S_{\omega, 1}\right\|_{C(\Pi)}<\frac{\varepsilon}{2}
$$

При $t \in\left[t_{0}, T\right]$ участок интегрирования $[0, t]$ разобьем на $m$ равных частей $\left[t_{j}, t_{j+1}\right)$, $j=0, \ldots, m-1$, и воспользуемся соотношением

$$
\begin{gathered}
S_{\omega, 1}=\sum_{n=1}^{n_{0}} f_{n} \sin n x \int_{0}^{t} \sin n(t-s) r_{1}(s, \omega s) d s \\
=\sum_{n=1}^{n_{0}} f_{n} \sin n x \sum_{j=0}^{m-1}\left[\int_{t_{j}}^{t_{j+1}} \sin n(t-s) r_{1}(s, \omega s) d s-\int_{t_{j}}^{t_{j+1}} \sin n\left(t-t_{j}\right) r_{1}\left(t_{j}, \omega s\right) d s\right] \\
+\sum_{n=1}^{n_{0}} f_{n} \sin n x \sum_{j=0}^{m-1} \int_{t_{j}}^{t_{j+1}} \sin n\left(t-t_{j}\right) r_{1}\left(t_{j}, \omega s\right) d s=U_{\omega, 1}+U_{\omega, 2} .
\end{gathered}
$$

Как и при доказательстве теоремы 1 выберем $m$ столь большим, что при всех $(x, t) \in$ $[0, \pi] \times\left[t_{0}, T\right]$ и $\omega>0$

$$
\left|U_{\omega, 1}\right|<\frac{\varepsilon}{4}
$$

В силу равенства $\left\langle r_{1}(t, \tau)\right\rangle_{\tau}=0$ подберем $\omega_{0}$ столь большим, что при выбранных $m, t \in\left[t_{0}, T\right]$ и всех $\omega>\omega_{0}$

$$
\left|U_{\omega, 1}\right|<\frac{\varepsilon}{4}
$$

В силу неравенств $(42),(43)$ существует такое число $\omega_{0}>0$, что при $\omega>\omega_{0}$

$$
\left|S_{\omega, 1}\right|<\frac{\varepsilon}{2} .
$$

Согласно соотношениям (40), (44) теорема 3 доказана. $\triangleright$

$\triangleleft$ ДоказАтельСтво ЛЕммы 1 . Пусть существует $t_{0} \in(0, T]$ такое, что $\left|r_{0}\left(t_{0}\right)\right|>$ $\left|r_{0}(0)\right|$. Воспользуемся представлением

$$
\Lambda_{n}\left(t_{0}\right)=\int_{0}^{t_{0}} \frac{\sin n\left(t_{0}-s\right)}{n} r_{0}(s) d s=\frac{r_{0}\left(t_{0}\right)-r_{0}(0) \cos n t_{0}}{n^{2}}+\int_{0}^{t_{0}} \frac{\cos n\left(t_{0}-s\right)}{n^{2}} r_{0}^{\prime}(s) d s .
$$

Отсюда видно, что найдутся положительные числа $c_{1}$ и $N_{1}$ такие, что при $n>\mathbb{N}$

$$
\left|\Lambda_{n}\left(t_{0}\right)\right|>\frac{c_{1}}{n^{2}} .
$$


Пусть теперь $r_{0}=$ const $\neq 0$. Без нарушения общности можем считать $r_{0}=1$. Тогда

$$
\Lambda_{n}\left(t_{0,1}\right)=\int_{0}^{t_{0,1}} \frac{\sin n\left(t_{0,1}-s\right)}{n} d s=\frac{1-\cos n t_{0,1}}{n^{2}}, \quad t_{0,1}=2 \pi \frac{l_{0}}{m_{0}}
$$

так что имеет место указанное в лемме заключение. Лемма 1 доказана. $\triangleright$

$\triangleleft$ ДокаЗАТЕЛЬСТво теоремЫ 4 . Пусть существует число $t_{0} \in(0, T]$ такое, что $\left|r_{0}\left(t_{0}\right)\right|>\left|r_{0}(0)\right|$. Предположим, что функция $f$, удовлетворяющая условиям п. 2.2, найдена. Тогда в силу теоремы 3 и соотношений (37), (39) справедливо равенство

$$
\sum_{n=1}^{\infty} f_{n} \Lambda_{n} \sin n x=\sum_{n=1}^{\infty} \psi_{n} \sin n x
$$

в котором коэффициенты $\psi_{n}$ представим в виде $\psi_{n}=\alpha_{n} n^{-5}$, где последовательность $\alpha_{n} \in l_{2}$. При $M_{0}=\emptyset$ из (45) с учетом леммы 1 однозначно находим

$$
f_{n}=\frac{\psi_{n}}{\Lambda_{n}}=\beta_{n} n^{-3}, \quad \beta_{n} \in l_{2} .
$$

Предполагая теперь, что функция $f$ не известна, восстановим ее по формуле

$$
f(x)=\sum_{n=1}^{\infty} f_{n} \sin n x
$$

где числа $f_{n}$ определяются соотношениями (46). При этом построенная функция $f$ будет, очевидно, удовлетворять требованиям определения обратной задачи 2 . Если $M_{0} \neq \emptyset$, то для возможности восстановления функции $f$, очевидно, необходимо и достаточно, чтобы при $n \in M_{0}$ выполнялись равенства $\psi_{n}=0$. В этом случае $f_{n}=0, n \in M_{0}$ и $f_{n}=\frac{\psi_{n}}{\Lambda_{n}}, n \notin M_{0}$. Таким образом доказана первая часть теоремы. Вторая часть (при $r_{0}=$ const $\neq 0$ ) доказывается аналогично. $\triangleright$

\section{3. Известно лишь среднее значение зависящего от времени сомножителя источника}

3.1. Обратная задача 3. В этом разделе вновь рассмотрим задачу вида (1)-(3) с $f(x, t) \equiv f(x) \in C^{4}([0, \pi]), f^{(2 k)}(0)=f^{(2 k)}(\pi)=0, k=0,1$, и функцией $r$ класса (A), удовлетворяющей дополнительному условию $r_{0} \in C^{2}([0, \pi])$. Будем считать, что функция $r_{0}$ известна, причем, существует такая точка $t_{0} \in(0, T]$, что $\left|r_{0}\left(t_{0}\right)\right|>\left|r_{0}(0)\right|$ (также нетрудно рассмотреть случай $r_{0}(t) \equiv$ const - см. раздел 2$)$, а функции $f$ и $r_{1}$ неизвестны. Пусть $\Lambda_{n}=\Lambda_{n}\left(t_{0}\right), n \in \mathbb{N},-$ набор чисел, вычисленных в соответствие с формулой (37). Ради краткости будем считать, что множество $M_{0}$ номеров $n$, при которых $\Lambda_{n}\left(t_{0}\right)=0$, пусто. Пусть задана в $Q$ непрерывная $2 \pi$-периодическая с нулевым средним по второй переменной функция $\chi(t, \tau)$, для которой определены производные $\frac{\partial^{k+2} \chi}{\partial t^{k} \partial \tau^{2}}, k=0,1,2,3$, принадлежащие $C(Q)$, а также функция $\psi \in C^{7}([0, \pi]), \psi^{(2 j)}(0)=\psi^{(2 j)}(\pi)=0, j=0,1,2,3$, и пусть существует точка $x_{0} \in(0, \pi)$, в которой $\widetilde{f}\left(x_{0}\right) \neq 0$, где

$$
\widetilde{f}(x)=\sum_{n=1}^{\infty} \widetilde{f}_{n} \sin n x, \quad \widetilde{f}_{n}=\frac{\psi_{n}}{\Lambda_{n}} .
$$


Как и в предыдущих пунктах рассмотрим функции $\varphi_{0}(t), \varphi_{1}(t), \varphi_{2}(t)$, определяющиеся следующим образом. Функция $\varphi_{0}(t)$ является решением задачи Коши:

$$
\left\{\begin{array}{l}
\varphi_{0}^{\prime \prime}(t)=\widetilde{f}\left(x_{0}\right) r_{0}(t)+\int_{0}^{t} K(t, s) r_{0}(s) d s \\
\varphi_{0}(0)=\varphi_{0}^{\prime}(0)=0
\end{array}\right.
$$

где

$$
K(t, s)=-\sum_{n=1}^{\infty} n \widetilde{f}_{n} \sin n(t-s) \sin n x_{0}
$$

Функции $\varphi_{1}, \varphi_{2}$ удовлетворяют условиям п.1.2 с заменой $f(x, t)$ на $\widetilde{f}(x)$, т. е.

$$
\begin{gathered}
\varphi_{1}(t)=-\sum_{n=1}^{\infty} \widetilde{f}_{n} \rho_{0 t}^{\prime}(0,0) \sin n t \frac{\sin n x_{0}}{n}, \\
\varphi_{2}(t)=-\sum_{n=1}^{\infty} \widetilde{f}_{n} \rho_{0}(0,0) \cos n t \sin n x_{0}+\sum_{n=1}^{\infty} \widetilde{f}_{n} \rho_{0 t}^{\prime}(0,0) \sin n t \frac{\sin n x_{0}}{n} .
\end{gathered}
$$

Обратная задача 3 состоит в нахождении таких функций $f$ и $r$, удовлетворяющих указанным в начале этого пункта условиям, что для решения $u_{\omega}(x, t)$ задачи $(1)-(3)$ будут выполнены соотношения

$$
\begin{gathered}
\left\|u_{\omega}\left(x_{0}, t\right)-\left[\varphi_{0}(t)+\frac{1}{\omega} \varphi_{1}(t)+\frac{1}{\omega^{2}}\left(\varphi_{2}(t)+\chi(t, \omega t)\right)\right]\right\|_{C([0, T])}=o\left(\omega^{-2}\right), \\
\left\|u_{\omega}\left(x, t_{0}\right)-\psi(x)\right\|_{C([0, \pi])}=o(1), \quad \omega \rightarrow \infty .
\end{gathered}
$$

Из результатов разделов 1,2 вытекает следующая теорема.

Теорема 5. Пусть функции $r_{0}(t), \varphi_{0}(t), \psi(x), \chi(t, \tau)$ и точки $x_{0}, t_{0}$ удовлетворяют указанным в этом пункте условиям. Тогда обратная задача 3 однозначно разрешима, т. е. существует единственная (в указанных в п.3.1 классах) пара функций $f$ и $r_{1}$, при которых решение $u_{\omega}(x, t)$ задачи (1)-(3) при $f(x, t) \equiv f(x)$ удовлетворяет условиям (48) и (49). При этом функция $f(x)=\widetilde{f}(x)$ вычисляется по формуле (47), a $r_{1}(t, \tau)=\left(f\left(x_{0}\right)\right)^{-1} \frac{\partial^{2}}{\partial \tau^{2}} \chi(t, \tau)$.

\section{4. Не известны оба сомножителя источника}

4.1. Обратная задача 4. Рассмотрим задачу (1)-(3), в которой функции $f(x, t) \equiv$ $f(x)$ и $r(t, \tau)$ не известны. Однако известно, что $r(t, \tau)-$ функция класса $(\mathrm{A})$ и дополнительно $r_{0} \in C^{2}([0, \pi])$, а $f(x)=\sum_{n=1}^{N} f_{n} \sin n x-$ функция с заданным числом $N$ гармоник с неизвестными амплитудами $f_{n}$. Пусть, кроме того, заданы точки $t_{0} \in(0, T)$, $x_{j} \in(0, \pi), j=0, \ldots, N-1$, где $x_{i} \neq x_{k}$ при $i \neq k$, а также функции $\varphi_{0}(t), \chi(t, \tau)$ и $\alpha_{j}(t)$, удовлетворяющие следующим условиям: $\varphi_{0}$ и $\chi-$ те же, что в п.1.2, и дополнительно $\varphi_{0} \in C^{4}([0, T)$,

$\alpha_{j} \in C^{1}\left(\left[t_{0}-\delta, t_{0}+\delta\right]\right)$ при некотором $\delta>0,\left(t_{0}-\delta, t_{0}+\delta\right) \subset(0, T), \quad j=1, \ldots, N-1$.

Введем еще функции:

$$
\varphi_{1}(t)=-\sum_{n=1}^{N} f_{n} \rho_{0 t}^{\prime}(0,0) \sin n t \frac{\sin n x}{n},
$$




$$
\varphi_{2}(t)=-\sum_{n=1}^{N} f_{n} \rho_{0}(0,0) \cos n t \sin n x+\sum_{n=1}^{N} f_{n} \rho_{0 t}^{\prime}(0,0) \sin n t \frac{\sin n x}{n},
$$

в которых фигурирует пока неизвестная функция $f(x)$.

Обратная задача 4 состоит в определении таких функций $f(x)$ и $r(t, \tau)$, удовлетворяющих указанным в начале этого пункта условиям, при которых для решения $u_{\omega}(x, t)$ задачи (1)-(3) выполнены асимптотические формулы

$$
\begin{gathered}
\left\|u_{\omega}\left(x_{0}, t\right)-\left[\varphi_{0}(t)+\frac{1}{\omega} \varphi_{1}(t)+\frac{1}{\omega^{2}}\left(\varphi_{2}(t)+\chi(t, \omega t)\right)\right]\right\|_{C([0, T])}=o\left(\omega^{-2}\right), \quad \omega \rightarrow \infty . \\
\left\|u_{\omega}\left(x_{j}, t\right)-\alpha_{j}(t)\right\|_{C\left(\left[t_{0}-\delta, t_{0}+\delta\right]\right)}=o(1), \quad j=1, \ldots, N-1, \omega \rightarrow \infty .
\end{gathered}
$$

Прежде чем изложить основной результат данного параграфа введем ряд обозначений и сформулируем некоторые дополнительные условия. Рассмотрим систему уравнений

$$
\left\{\begin{array}{l}
\sum_{n=1}^{N} \psi_{n} \sin n x_{0}=\varphi_{0}\left(t_{0}\right) \\
\sum_{n=1}^{N} \psi_{n} \sin n x_{j}=\alpha_{j}\left(t_{0}\right), \quad j=1, \ldots, N-1,
\end{array}\right.
$$

относительно неизвестных $\psi_{n}, n=1, \ldots, N$. Поскольку матрица $A=\left(\sin n x_{j}\right)_{n=1, j=0}^{N, N-1}$ невырождена, то из (54) однозначно найдем вектор-функцию $\psi \equiv \psi\left(\varphi_{0}\left(t_{0}\right), \alpha\left(t_{0}\right)\right)^{* *}$. Нормируем искомую функцию $r_{0}(t)$ условием $r_{0}\left(t_{0}\right)=1$. Рассмотрим теперь систему

$$
\left\{\begin{array}{l}
\sum_{n=1}^{N} f_{n} \sin n x_{0}=\sum_{n=1}^{N} n^{2} \psi_{n} \sin n x_{0}+\varphi_{0}^{\prime \prime}\left(t_{0}\right) ; \\
\sum_{n=1}^{N} f_{n} \sin n x_{j}=\sum_{n=1}^{N} n^{2} \psi_{n} \sin n x_{j}+\alpha_{j}^{\prime \prime}\left(t_{0}\right), \quad j=1, \ldots, N-1,
\end{array}\right.
$$

относительно неизвестных $f_{n}, n=1, \ldots, N$, из которой однозначно найдем вектор

$$
f \equiv F\left(\psi\left(\varphi_{0}\left(t_{0}\right), \alpha\left(t_{0}\right)\right), \varphi_{0}^{\prime \prime}\left(t_{0}\right), \alpha^{\prime \prime}\left(t_{0}\right)\right)=f\left(\varphi_{0}, \alpha, t_{0}\right) .
$$

Будем предполагать, что выполнено соотношение

$$
\sum_{n=1}^{N} f_{n} \sin n x_{0} \neq 0
$$

Рассмотрим теперь уравнение Вольтерра второго рода

$$
f\left(x_{0}\right) l(t)+\int_{0}^{t} K(t, s) l(s) d s=\mu(t), \quad \mu \in C([0, T])
$$

где $K(t, s)=-\sum_{n=1}^{N} n f_{n} \sin n(t-s) \sin n x_{0}, f(x)=\sum_{n=1}^{N} f_{n} \sin n x, f_{n}$ определены в (56). Его единственное в пространстве $C([0, T])$ решение $l(t)$ обозначим символом $S\left(\varphi_{0}, \alpha, t_{0}, \mu(t)\right)$.

**Зависимость функций от точек $x_{j}, j=0, \ldots, N-1$, мы здесь и ниже для упрощения записи не отмечаем. 
Теорема 6. Для любого набора функций $\varphi_{0}, \chi, \alpha_{j}, j=1, \ldots, N-1$, и точек $t_{0}$, $x_{k}, k=0, \ldots, N-1$, удовлетворяющих указанным выше условиям, обратная задача 4 однозначно разрешима тогда и только тогда, когда выполнены следующие условия согласования:

$$
\begin{gathered}
\sum_{n=1}^{N} \sin n x_{j} f_{n}\left(\varphi_{0}, \alpha, t_{0}\right) \int_{0}^{t} \frac{\sin n(t-s)}{n} S\left(\alpha, t_{0}, \varphi_{0}^{\prime \prime}(s)\right) d s=\alpha_{j}(t), \\
j=1, \ldots, N-1, \quad t \in\left(t_{0}-\delta, t_{0}+\delta\right) .
\end{gathered}
$$

ЗАмЕЧАНИЕ. При выполнении условий (59) для нахождения функции $f(x)$ требуется решить две системы линейных уравнений с единой невырожденной основной матрицей; для нахождения $r_{0}(t)$ - решить уравнение Вольтерра второго рода $(58)$ с $\mu(t)=\varphi_{0}^{\prime \prime}(t)$, а функция $r_{1}(t, \tau)=\left(f\left(x_{0}\right)\right)^{-1} \frac{\partial^{2}}{\partial \tau^{2}} \chi(t, \tau)$.

$\triangleleft$ ДокаЗАТЕЛЬСТво тЕОРЕмЫ 6 . Предположим, что пара функций $(f, r)$ является решением обратной задачи. Как и при доказательстве теоремы 2 представим решение $u_{\omega}(x, t)$ в виде (8)-(13). Таким образом в силу теорем 1 и 3 , а также соотношений (52), (53) получим систему

$$
\left\{\begin{array}{l}
{\left[u_{0}+\omega^{-1} u_{1}+\omega^{-2}\left[u_{2}+v_{2}\right]\right]_{x=x_{0}}=\varphi_{0}+\frac{1}{\omega} \varphi_{1}+\frac{1}{\omega^{2}}\left[\varphi_{2}+\chi\right]+o\left(\omega^{-2}\right) ;} \\
\left.u_{0}\right|_{x=x_{j}}=\alpha_{j}, \quad j=1, \ldots, N-1 .
\end{array}\right.
$$

Приравнивая в первом уравнении (60) коэффициенты при $\omega^{0}$, и учитывая представление (37) функции $u_{0}$, придет к системе

$$
\left\{\begin{array}{l}
\sum_{n=1}^{N} f_{n} \Lambda_{n}(t) \sin n x_{0}=\varphi_{0}(t) ; \\
\sum_{n=1}^{N} f_{n} \Lambda_{n}(t) \sin n x_{j}=\alpha_{j}(t), \quad j=1, \ldots, N-1, t \in\left[t_{0}-\delta, t_{0}+\delta\right] .
\end{array}\right.
$$

Полагая в уравнениях последней системы $t=t_{0}, f_{n} \Lambda_{n}\left(t_{0}\right)=\psi_{n}$, придем к системе (54). Как было отмечено выше, вектор $\psi$ находится отсюда однозначно. Продифференцировав уравнения (61) дважды по $t$ и вновь подставляя $t=t_{0}, f_{n} \Lambda_{n}\left(t_{0}\right)=\psi_{n}$, получим систему (55), откуда находим вектор $f$. Вопрос о нахождении функции $r$ при известной $f(x)$ исследован в теореме 2. Таким образом, нахождение функции $r_{0}(t)=S\left(\varphi_{0}, \alpha, t_{0}, \varphi_{0}^{\prime \prime}(t)\right)$ сведено к решению уравнения Вольтерра второго рода вида (58); а также $r_{1}(t, \tau)=$ $\left(f\left(x_{0}\right)\right)^{-1} \frac{\partial^{2}}{\partial \tau^{2}} \chi(t, \tau)$. Из проведенных рассуждений следует, что если обратная задача 4 разрешима, то она разрешима однозначно и в силу требования (53) условие согласования (59) выполнено.

Пусть теперь известно, что условие (59) выполнено. Определим $f(x)$ и $r(t, \tau)$ формулами, которые установлены в первой части доказательства теоремы. Тогда для них справедливы теорема 1 и решение $u_{\omega}$ задачи (1)-(3) представимо в виде (8)-(13), где

$$
\begin{gathered}
u_{0}(x, t)=\sum_{n=1}^{N} f_{n}\left(\varphi_{0}, \alpha, t_{0}\right) \sin n x \int_{0}^{t} \frac{\sin n(t-s)}{n} S\left(\varphi_{0}, \alpha, t_{0}, \varphi_{0}^{\prime \prime}(s)\right) d s, \\
u_{1}(x, t)=-\sum_{n=1}^{N} f_{n}\left(\varphi_{0}, \alpha, t_{0}\right) \rho_{0 t}^{\prime}(0,0) \frac{\sin n x}{n} \sin n t
\end{gathered}
$$




$$
\begin{gathered}
u_{2}(x, t)=-\sum_{n=1}^{N} f_{n}\left(\varphi_{0}, \alpha, t_{0}\right) \rho_{0}(0,0) \cos n t \sin n x+\sum_{n=1}^{N} f_{n}\left(\varphi_{0}, \alpha, t_{0}\right) \rho_{0 t}^{\prime} \sin n t \frac{\sin n x}{n}, \\
v_{2}(x, t)=\sum_{n=1}^{N} f_{n}\left(\varphi_{0}, \alpha, t_{0}\right) \sin n x \rho_{0}(t, \tau) .
\end{gathered}
$$

Покажем, что для функции $u_{\omega}$ будут выполнены условия (52), (53). Для этого, в силу теоремы 1, достаточно доказать равенства:

$$
\begin{gathered}
u_{0}\left(x_{0}, t\right)=\varphi_{0}(t), \quad u_{1}\left(x_{0}, t\right)=\varphi_{1}(t), \quad u_{2}\left(x_{0}, t\right)=\varphi_{2}(t), \\
v_{2}\left(x_{0}, t, \tau\right)=\chi(t, \tau), \quad u_{0}\left(x_{j}, t\right)=\alpha_{j}(t) .
\end{gathered}
$$

Справедливость первого из них нами установлена в ходе предыдущей части доказательства данной теоремы. Второе и третье равенства выполняются согласно представлениям (50), (51) функций $\varphi_{1}, \varphi_{2}$ и теоремы 1 . Четвертое равенство, очевидно, также справедливо. Последнее вытекает из условия согласования (59). Теорема 6 доказана. $\triangleright$

4.2. ПримеР. Рассмотрим обратную задачу (1)-(3) в случае $N=2$. В качестве исходных данных возьмем следующие:

$$
\begin{gathered}
t_{0}=1, x_{0}=\frac{\pi}{2}, x_{1}=\frac{\pi}{6}, \\
\varphi_{0}(t)=\sin t-t, \\
\alpha_{1}(t)=\frac{1}{2} \sin t-\frac{\sqrt{3}}{16} \sin 2 t-\frac{1}{2} t+\frac{\sqrt{3}}{8} t, \\
\chi(t, \tau)=\cos \tau .
\end{gathered}
$$

Проверим для этих данных справедливость условий теоремы 6 (59). Система (54) в данном случае принимает вид

$$
\left\{\begin{array}{l}
\psi_{1}=\sin 1-1 \\
\frac{1}{2} \psi_{1}+\frac{\sqrt{3}}{2} \psi_{2}=\frac{4 \sin 1-\frac{\sqrt{3}}{2} \sin 2-4+\sqrt{3}}{8}
\end{array}\right.
$$

откуда находим $\psi_{1}=\sin 1-1, \psi_{2}=-\frac{1}{8} \sin 2+\frac{1}{4}$. Система (55) имеет вид

$$
\left\{\begin{array}{l}
f_{1}=\sin 1-1-\sin 1 \\
\frac{1}{2} f_{1}+\frac{\sqrt{3}}{2} f_{2}=-\frac{1}{2}+2 \sqrt{3}\left(-\frac{1}{8} \sin 2+\frac{1}{4}\right)+\frac{\sqrt{3}}{4} \sin 2
\end{array}\right.
$$

откуда определим $f_{1}=-1, f_{2}=1$. Справедливо соотношение $(57):-\sin \frac{\pi}{2}+\sin \pi=-1 \neq$ 0. Из уравнения Вольтерра второго рода

$$
-r_{0}(t)+\int_{0}^{t} \sin (t-s) r_{0}(s) d s=-\sin t
$$

находим $r_{0}(t)=t$. Теперь можно выписать условие согласования (59):

$$
-\frac{1}{2} \int_{0}^{t} s \sin (t-s) d s+\frac{\sqrt{3}}{4} \int_{0}^{t} s \sin 2(t-s) d s=\alpha_{1}(t), \quad t \in\left(t_{0}-\delta, t_{0}+\delta\right), \delta>0 .
$$

Легко проверяется его справедливость. Таким образом

$$
f(x)=-\sin x+\sin 2 x, \quad r_{0}(t)=t, \quad r_{1}(t, \tau)=\cos \tau .
$$




\title{
Литература
}

1. Babich P. V., Levenshtam V. B. Direct and inverse asymptotic problems high-frequency terms // Asymptotic Analysis.-2016.--Vol. 97.-P. 329-336.

2. Бабич П. В., Левенштам В. Б., Прика С. П. Восстановление быстро осциллирующего источника в уравнении теплопроводности по асимптотике решения // Журн. вычисл. матем. и матем. физ.2017.-Т. 57, № 12.-С. 1955-1965. DOI: 10.7868/S0044466917120079.

3. Лаврентьев М. М., Резницкая К. Г., Яхно В. Г. Одномерные обратные задачи математической физики.-Новосибирск: Наука, 1982.-88 с.

4. Романов В. Г. Обратные задачи математической физики.-М.: МГУ, 1984.

5. Денисов А. М. Введение в теорию обратных задач.-М.: Наука, 1994.

6. Кабанихин С. И. Обратные и некорректные задачи.-Новосибирск: Сиб. науч. изд-во, 2008. -450 с.

7. Денисов А. М. Асимптотика решений обратных задач для гиперболического уравнения с малым параметром при старшей производной // Журн. вычисл. матем. и матем. физ.-2013.-Т. 53, № 5.C. 744-752. DOI: $10.7868 / \mathrm{S} 0044466913050049$.

8. Денисов А. М. Задачи определения неизвестного источника в параболическом и гиперболическом уравнениях // Журн. вычисл. матем. и матем. физ.-2015.-Т. 55, № 5.-С. 830-835. DOI: 10.7868/S0044466915050087.

9. Камынин В. Л. Обратная задача одновременного определения правой части и коэффициента при младшей производной в параболическом уравнении на плоскости // Диф. уравнения.-2014.-T. 50, № 6.-С. $795-806$.

10. Бабич П. В., Левенштам В. Б. Восстановление быстро осциллирующего свободного члена в многомерном гиперболическом уравнении // Мат. заметки.-2018.-T. 104, № 4.-C. 505-515. DOI: $10.4213 / \operatorname{mzm} 12151$.

11. Ильин В. А. О разрешимости смешанных задач для гиперболического и параболического уравнений // Успехи мат. наук.-1960.-Т. 15, № 2.-С. 97-154.

Статъя поступила 8 августа 2020 г.

БАБИЧ ПАВЕЛ ВАСИЛЬЕВИЧ

Математический институт им. В. А. Стеклова,

младший научный сотрудник

РОССИЯ, 119991, Москва, ул. Губкина, 8

E-mail: xblahblahc@gmail.com

ЛЕВЕНШТАМ ВАЛЕРИЙ БОРИСОВИЧ

Математический институт им. В. А. Стеклова,

ведущий научный сотрудник

РОССИЯ, 119991, Москва, ул. Губкина, 8

E-mail: vlevenshtam@yandex.ru

Vladikavkaz Mathematical Journal

2020, Volume 22, Issue 4, P. 28-44

\section{RECOVERY OF RAPIDLY OSCILLATED RIGHT-HAND SIDE OF THE WAVE EQUATION BY THE PARTIAL ASYMPTOTICS OF THE SOLUTION}

\author{
Babich, P. V. ${ }^{1}$ and Levenshtam, V. B. ${ }^{1}$ \\ ${ }^{1}$ Steklov Mathematical Institute of RAS, \\ 8 Gubkina St., Moscow 119991, Russia \\ E-mail: xblahblahc@gmail.com, vlevenshtam@yandex.ru
}

\begin{abstract}
The initial-boundary problem for the one-dimensional wave equation with unknown rapidly oscillated right-handside is considered in the paper. The latter is represented as a product of two functions; the first function depends on the spatial variable and the second one depends on time and fast time variables.
\end{abstract}


Four different cases are considered: in two cases one of the factor-functions is known and in two other cases both factor-functions are unknown. In each of these cases, the inverse problems of recovering unknown functions from some information about partial asymptotics of the original problem with known data are posed and solved. This specified information consists, in general, in setting values for certain asymptotics coefficients in some spatial and/or time points. The use of some additional conditions (overdetermination conditions) in this form speaks of a fundamental difference between the above statements of inverse problems and the classics, where additional conditions are imposed on exact solutions. The construction of solutions asymptotics of the original problem with this approach act as direct problem. This approach to inverse problems with rapidly oscillated data in time is developed by the author over the past few years.

Key words: one-dimensional wave equation, rapidly oscillating absolute term, asymptotics of solution, inverse problem.

Mathematical Subject Classification (2010): 35L05, 35L20, 35R30.

For citation: Babich, P. V. and Levenshtam, V. B. Recovery of Rapidly Oscillated Right-Hand Side of the Wave Equation by the Partial Asymptotics of the Solution, Vladikavkaz Math. J., 2020, vol. 22, no. 4, pp. 28-44 (in Russian). DOI: 10.46698/s0301-1959-8380-s.

\section{References}

1. Babich, P. V. and Levenshtam, V. B. Direct and Inverse Asymptotic Problems High-frequency Terms, Asymptotic Analysis, 2016, vol. 97, pp. 329-336.

2. Babich, P. V., Levenshtam, V. B. and Prika, S. P. Recovery of a Rapidly Oscillating Source in the Heat Equation from Solution Asymptotics, Computational Mathematics and Mathematical Physics, 2017, vol. 57, no. 12 , pp. 1908-1918. DOI: 10.1134/S0965542517120065.

3. Lavret'ev, M. M., Reznitskaya, K. G. and Yakhno, V. G. Odnomernye obratnye zadachi matematicheskoj fiziki [One-Dimensional Inverse Problems of Mathematical Physics], Novosibirsk, Nauka, 1982 (in Russian).

4. Romanov, V. G. Obratnye zadachi matematicheskoj fiziki [Inverse Problems of Mathematical Physics], Moscow, Nauka, 1984 (in Russian).

5. Denisov, A. M. Vvedenie v teoriyu obratnyh zadach [Introduction to the Theory of Inverse Problems], Moscow, Nauka, 1994 (in Russian).

6. Kabanikhin, S. I. Obratnye i nekorrektnye zadachi [Inverse and Ill-Posed Problems], Novosibirsk, Sibirskoe Nauchnoe Izdatel'stvo, 2008 (in Russian).

7. Denisov, A. M. Asymptotic Expansions of Solutions to Inverse Problems for a Hyperbolic Equation with a Small Parameter Multiplying the Highest Derivative, Computational Mathematics and Mathematical Physics, 2013, vol. 53, no. 5, pp. 580-587. DOI: 10.1134/S0965542513050047.

8. Denisov, A. M. Problems of Determining the Unknown Source in Parabolic and Hyperbolic Equations, Computational Mathematics and Mathematical Physics, 2015, vol. 55, no. 5, pp. 829-833. DOI: 10.1134/S0965542515050085.

9. Kamynin, V. L. Inverse Problem of Simultaneously Determining the Right-hand Side and the Coefficient of a Lower Order Derivative for a Parabolic Equation on the Plane, Differential Equations, 2014, vol. 50, no. 6, pp. $792-804$.

10. Babich, P. V. and Levenshtam, V. B. Recovery of a Rapidly Oscillating Absolute Term in the Multidimensional Hyperbolic Equation. Mathematical Notes, 2018, vol. 104, no. 4, pp. 489-497. DOI: 10.1134/S000143461809016X.

11. Ilin, V. A. The Solvability of Mixed Problems for Hyperbolic and Parabolic Equations, Russian Mathematical Surveys, 1960, vol. 15, no. 1, pp. 85-142. DOI: 10.1070/RM1960v015n02ABEH004217.

Received August 8, 2020

PAVEl V. BABich

Steklov Mathematical Institute of RAS,

8 Gubkina St., Moscow 119991, Russia,

Junior Researcher

E-mail: xblahblahc@gmail.com

VAleriy B. Levenshtam

Steklov Mathematical Institute of RAS,

8 Gubkina St., Moscow 119991, Russia,

Leading Researcher

E-mail: vlevenshtam@yandex.ru 